\title{
The Relationship between Neck and Shoulder Pain and the Sagittal Alignment of the Spine in Standing in Younger Generation
}

\author{
Tomonori Kenmoku1,2*, Takane Suzuki ${ }^{3}$, Nobuyasu Ochiai ${ }^{4}$, Tatsuki Ebata², \\ Genyo Miyajima ${ }^{5}$, Naoshige Nagura ${ }^{1}$, Mitsufumi Nakawaki', Ryo Tazawa ${ }^{1}$, Sho Hiramatsu', \\ Hironori Yamazaki ${ }^{4}$, Yu Sasaki ${ }^{4}$, Takeshi Yamaguchi', Eiko Hashimoto4, Kenji Onuma1, \\ Naonobu Takahira ${ }^{6}$, Kazuhisa Takahashi $^{4}$, Masashi Takaso ${ }^{1}$ \\ ${ }^{1}$ Department of Orthopedic Surgery, Kitasato University School of Medicine, Sagamihara, Japan \\ ${ }^{2}$ Sakura Orthopaedic Hospital, Sakura, Japan \\ ${ }^{3}$ Department of Bioenvironmental Medicine, Graduate School of Medicine, Chiba, Japan \\ ${ }^{4}$ Department of Orthopaedic Surgery, Chiba University Graduate School of Medicine, Chiba, Japan \\ ${ }^{5}$ Department of Orthopedic Surgery, Kitasato Medical Center, Kitamoto, Japan \\ ${ }^{6}$ School of Allied Health Science, Kitasato University, Sagamihara, Japan \\ Email: *pseudolefty811@yahoo.co.jp
}

Received 21 September 2015; accepted 31 October 2015; published 3 November 2015

Copyright (C) 2015 by authors and Scientific Research Publishing Inc.

This work is licensed under the Creative Commons Attribution International License (CC BY).

http://creativecommons.org/licenses/by/4.0/

(c) (i) Open Access

\section{Abstract}

Background: The relationship between chronic neck and shoulder pain and posture remains controversial. The purpose of this study was to investigate the relationship between chronic neck and shoulder pain and spinal sagittal alignment in standing posture in younger generation. Methods: Subjects included 57 females and 32 males (average age, $29.9 \pm 5.7$ years). All subjects were 20s or 30s. Spinal curvature was assessed using SpinalMouse. The subjects were also divided into a normal group (VAS zero group) and a pain group by VAS results. Statistical analysis was performed by Student's t-test. Significance was defined as $p<0.05$. Results: The normal group and pain group included 29 and 60 subjects, respectively. In terms of location of pain, thirty-one subjects felt neck pain, 50 felt pain above the scapula, and 17 felt pain between the thoracic spine and scapula. Thoracic kyphosis and lumbar lordosis in the pain group were significantly higher than those in the normal group ( $p=0.013$ and $p=0.020$, respectively). Thoracic kyphosis in subjects with neck pain or pain above scapula was significantly higher than that in subjects without pain $(p=0.0075$ and $p$ $=0.025$, respectively). Lumbar lordosis in subjects with pain above the scapula or interscapula was significantly higher than that in subjects without pain $(p=0.016)$.

\footnotetext{
"Corresponding author.
}

How to cite this paper: Kenmoku, T., et al. (2015) The Relationship between Neck and Shoulder Pain and the Sagittal Alignment of the Spine in Standing in Younger Generation. Open Journal of Orthopedics, 5, 337-344. 


\section{Keywords}

\section{Choronic Neck and Shoulder Pain, Sagittal Alignment of the Spine, Thoracic Kyphosis, Lumber Lordosis}

\section{Introduction}

Chronic neck and shoulder pain is one of major muscloskeltal disorders, and not only elder generation, but younger generation suffer from this disorder. Chronic neck and shoulder pain is one of the most common conditions in our country [1]. The condition is called "Katakori" in Japanese. This condition is defined as pain, lassitude and pain around the neck and shoulders. Many factors, including cervical radiculopathy and psychological factors, are suggested to be involved in chronic neck and shoulder pain [2]-[7]. These multiple factors make understanding this disease increasingly complex. A characteristic of this disease is that both young and old individuals are affected; thus, something other than age must have a significant impact on its development. The sagittal alignment of the spine is thought to be one of the most important factors that influence disorders around the neck and shoulder [8]-[12]. The reciprocal curvature of the spine in sagittal alignment allows for efficient absorption of the loads applied to the spinal column and increases the efficiency of the spinal musculature [13]. A slouched posture significantly reduces scapular upward rotation in arm elevation [10] [12] [14] [15]. However, there are no reports concerning the relationship between chronic neck and shoulder pain and spinal alignment in younger individuals. The purpose of this study was to investigate the relationship between chronic neck and shoulder pain and the sagittal alignment of the spine in a standing posture before the appearance of apparent degenerative change in spinal alignment.

\section{Materials and Methods}

A total of 89 subjects (57 females and 32 males) agreed to participate in this study. All were volunteers and did not meet the following exclusion criteria: limitation of passive range of motion in the shoulder, symptoms of cervical radiculopathy, and history of injury around the shoulder and spine detected prior to or during this study. All volunteers provided informed consent. All participants were healthy adults in their in 20s or 30s. The average age at the time of the investigation was $29.9 \pm 5.7$ years.

Chronic neck and shoulder pain was assessed by the visual analogue scale (VAS), and the location of pain was identified. The sagittal alignment of the spine was measured by the SpinalMouse (Idiag, Volketswil, Switzerland) in a standing posture.

All subjects rated the VAS score of pain from 0 to 100, with 0 indicating no pain and 100 indicating the worst possible pain. Subjects were divided into a normal group and pain group by VAS results. The VAS score of the normal group was 0 , and other results were included in the pain group. Subjects with a VAS score of 0 confirmed that they had not felt pain for $>1$ month. The location of pain was classified in accordance with the JOA Katakori project [16]. In this report, the location was divided into the following four places: neck, above the scapula, interscapular area, and on the scapula (Figure 1).

For each person, measures of sagittal spinal alignment were made using the SpinalMouse, a hand-held, computer-assisted electromechanical device that noninvasively measures sagittal spinal alignment and intersegmental angles. Many authors have reported the reliability of assessment of sagittal alignment of the spine with the use of the SpinalMouse [17]-[19]. The subject was asked to assume a natural standing position. The SpinalMouse was run paravertebrally along the spinal column from C7 to the rimaani (S3). This device is too big to measure the cervical curvature, therefore, we measured from C7. When manually guided paravertebrally along the spine of a subject, the system recorded the outline of the skin over the spinal column in the sagittal plane. The local angles and inclination relative to a perpendicular line were given by an internal pendulum connected to a potentiometer. Raw SpinalMouse measurement data were the superficial back length from C7 to S3 and the local angle of each point of this length relative to the plumb line. In this manner, 17 segments (Th1/2 to L5/S1) were evaluated; inclination of the spine, and the hip/sacral angle were calculated. In accordance with Vialle et al., we measured thoracic kyphosis angle (TK) and lumbar lordosis angle (LL) based on the graphic demonstration of the SpinalMouse [13]. TK was reported as the angle between the cranial end plate of T4 and the caudal end 


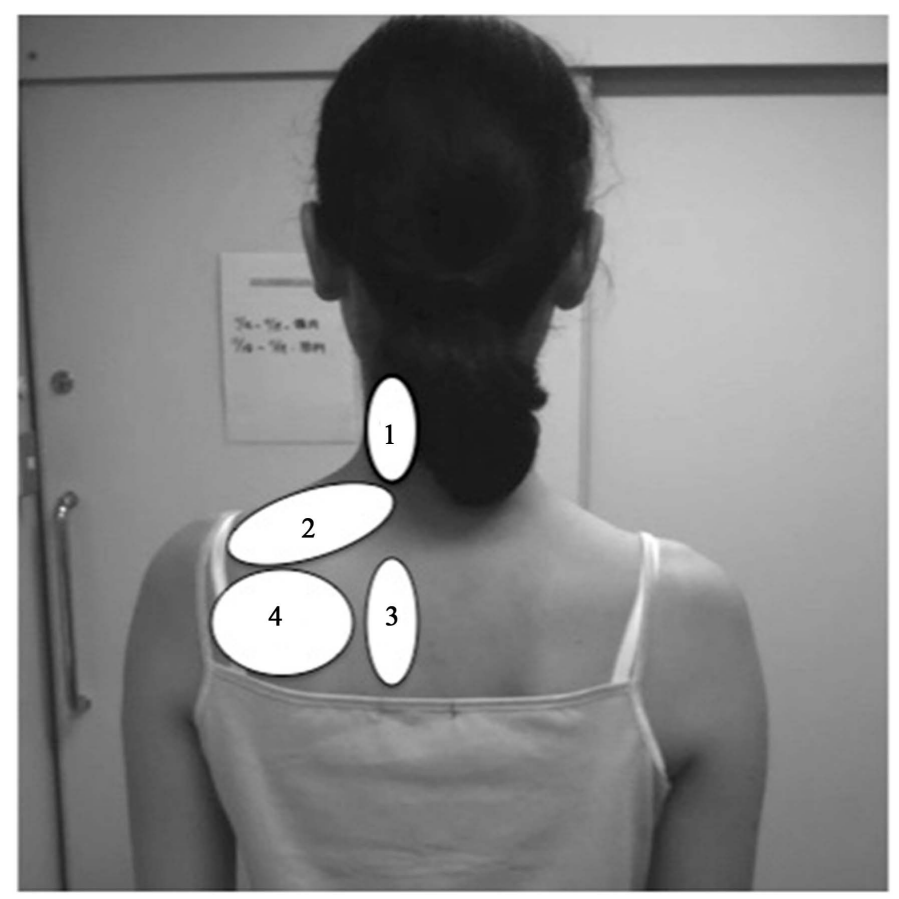

Figure 1. The location of neck and shoulder stiffness. The location of neck and shoulder stiffness was classified in accordance with the JOA Katakori project [21]. In this report, the location was divided into the following four places: 1 : neck, 2: above the scapula, 3: interscapular area, and 4: on the scapula.

plate of the transitional vertebra located at the junction of the thoracic kyphosis and lumbar lordosis. LL was the angle between the cranial end plate of the transitional vertebra and the cranial end plate of S1 [13]. These angles show the thoracic kyphosis and lumbar lordosis based on the junction of the thoracic and lumber spine. In this study, we drew the tangential line to upper thoracic line and the transitional line to measure TK, and drew the tangential line to the transitional line and L5/S1 to measure LL (Figure 2).

The following six angles were compared between the normal and pain groups and between genders: TK, LL, inclination, and hip/sacral angle. These six angles were also compared by gender and between groups and were divided by the result of VAS at each location of pain. The correlation between VAS and each angle was then assessed.

\subsection{Statistic Analysis}

Statistical analysis of results was performed using JMP software, version 9.0 (SAS Institute, Cary, NC, USA). Comparisons between two groups were performed by Student t-test. Significant differences were defined as $p<$ 0.05. Statistical power was analyzed using PS version 3.0.34 (Vanderbilt University, Nashville, TN, USA).

\subsection{Ethics Approval}

The protocols for this investigation were approved by the Ethics Committee of Sakuraorthopaedic hospital in 2010. The study number is 23-10. All authors, their immediate family, and any research foundation with which they are affiliated did not receive any financial payments or other benefits from any commercial entity related to the subject of this article.

\subsection{Competing Interests}

We wish to confirm that there are no known conflicts of interest associated with this publication. The Parents' Association of Kitasato University, School of Medicine for providing a grant to support this investigation. However, there has been no significant financial support for this work that could have influenced its outcome. 

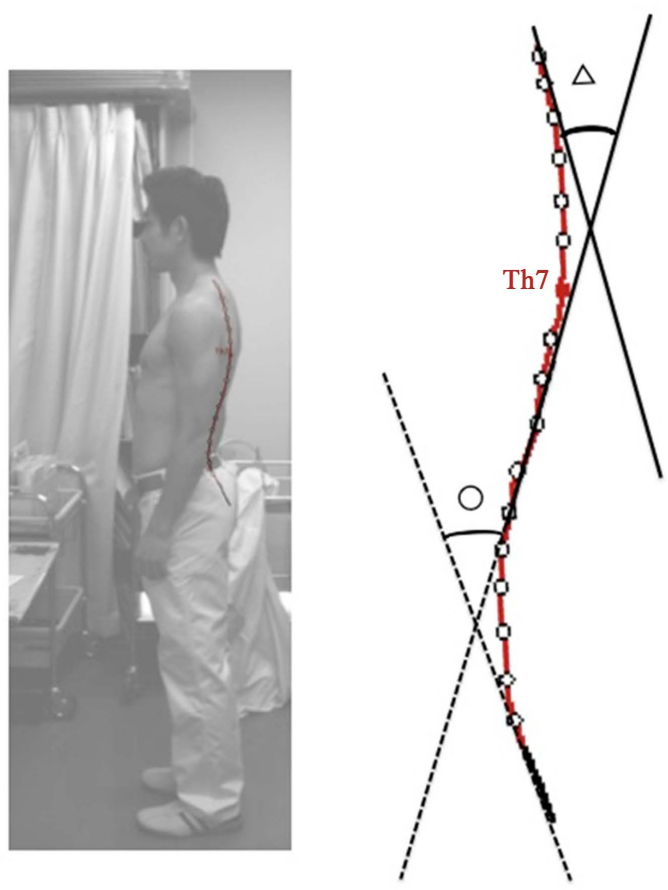

Figure 2. The angle of maximum thoracic kyphosis and maximum lumbar lordosis. $\triangle$ : maximum thoracic kyphosis, ०: maximum lumbar lordosis.

\section{Results}

The normal group comprised 29 subjects (12 females and 17 males), and the pain group comprised 60 subjects (45 females and 15 males). The average age in each group was $31.6 \pm 6.7$ and $29.1 \pm 5.0$ years, respectively. There was no significant difference in age $(p=0.10)$. Average VAS in the pain group was $53.7 \pm 24.4$. Table 1 shows details of each angle. The pain group showed significant increases in TK and LL ( $p=0.013, \beta=0.71$ and $p=0.020, \beta=0.65$ respectively). There was no significant difference in any angle between genders.

In terms of location of pain, 31 subjects felt pain in the neck ( 1 on the dominant side, 2 on the nondominant side, and 28 on both sides). A total of 50 subjects felt pain above the scapula ( 2 on the dominant side, 3 on nondominant side, and 45 on the both sides) A total of 17 subjects felt pain in the interscapular area ( 1 on the dominant side, 2 on the nondominant side, and 14 on both sides). One subjects felt pain of the scapula (1 on the nondominant side). Therefore, pain of the scapula was excluded from statistical analysis. Comparisons of each angle between the normal and pain groups at each location of pain are shown in Table 2. There was a significant difference in TK between normal group and subjects who felt pain in the neck ( $p=0.0075, \beta=0.78)$. In terms of pain above the scapula, TK and LL significantly increased $(p=0.025, \beta=0.62$ and $p=0.016, \beta=0.68$ respectively). There was a significant difference in LL between normal group and subjects who felt pain in the interscapular area $(p=0.032, \beta=0.58)$.

\section{Discussion}

Many papers have reported a relationship between posture and pain and pain around the neck and shoulders [8] [9] [11] [12] [15] [20] [21]. However, previous papers have assessed the alignment including in old generation, or have not reported in detail on the location of pain around the neck and shoulders. Ours was the first report to examine the relationship between sagittal spinal alignment and chronic neck and shoulder pain and to classify the location of the pain in younger individuals. Our results showed that thoracic kyphosis and lumbar lordosis were related to chronic neck and shoulder pain. Mean TK and mean LL of pain group were significantly larger than those of normal. In particular, in terms of neck pain and pain above the scapula, TK increased in subjects with symptoms significantly. Certain postures, such as a forward head posture and slouched position, induce an 
Table 1. (a) Angles between normal and pain group; (b) Angles between genders.

(a)

\begin{tabular}{cccc}
\hline & Normal group $(\mathrm{n}=29)$ & Pain group $(\mathrm{n}=60)$ & $p$ value $(\beta$ value $)$ \\
\hline TK & $27.0 \pm 5.0$ & $31.4 \pm 8.6$ & $0.013(0.71)$ \\
LL & $24.9 \pm 6.7$ & $28.8 \pm 7.5$ & $0.020(0.65)$ \\
Sacral/hip angle & $17.4 \pm 5.6$ & $18.0 \pm 5.3$ & 0.95 \\
Inclination & $3.9 \pm 2.5$ & $3.8 \pm 2.7$ & 0.97 \\
\hline
\end{tabular}

Table 1 showed each angle between normal and pain group, and each angle between genders. The pain group showed significant increases in TK and LL. TK and LL show the angles of thoracic kyphosis and umber lordosis which measured in accordance with Vialle et al. [13].

(b)

\begin{tabular}{cccc}
\hline & Female $(\mathrm{n}=57)$ & Male $(\mathrm{n}=32)$ & $p$ value \\
\hline TK & $30.1 \pm 8.4$ & $29.8 \pm 6.8$ & 0.89 \\
LL & $28.1 \pm 7.5$ & $26.6 \pm 7.3$ & 0.36 \\
Sacral/hip angle & $18.0 \pm 5.1$ & $17.4 \pm 5.9$ & 0.64 \\
Inclination & $3.6 \pm 2.8$ & $4.1 \pm 2.4$ & 0.40
\end{tabular}

There was no significant difference in any angle between genders. TK: Thoracic kyphosis LL: Lumber lordosis.

Table 2. (a) Angles between normal and neck pain group; (b) Angles between normal and pain above the scapula group; (c) Angles between normal and pain at the interscapula group.

(a)

\begin{tabular}{cccc}
\hline Neck pain & Normal group $(\mathrm{n}=29)$ & Pain group $(\mathrm{n}=31)$ & $p$ value $(\beta$ value $)$ \\
\hline TK & $27.0 \pm 5.0$ & $31.7 \pm 7.8$ & $0.0075(0.78)$ \\
LL & $24.9 \pm 6.7$ & $28.6 \pm 8.5$ & 0.069 \\
Sacral/hip angle & $17.4 \pm 5.6$ & $17.7 \pm 6.1$ & 0.72 \\
Inclination & $3.9 \pm 2.5$ & $4.1 \pm 2.8$ & 0.78 \\
\hline
\end{tabular}

Table 2(a) showed each angle between the subjects who felt neck pain and subjects without pain. There was a significant difference in TK between two groups. TK: Thoracic kyphosis LL: Lumber lordosis.

(b)

\begin{tabular}{cccc}
\hline Pain above the scapula & Normal group $(\mathrm{n}=29)$ & Pain group $(\mathrm{n}=50)$ & $p$ value $(\beta$ value $)$ \\
\hline TK & $27.0 \pm 5.0$ & $31.1 \pm 8.8$ & $0.025(0.62)$ \\
LL & $24.9 \pm 6.7$ & $29.1 \pm 7.5$ & $0.016(0.68)$ \\
Sacral/hip angle & $17.4 \pm 5.6$ & $18.3 \pm 4.9$ & 0.64 \\
Inclination & $3.9 \pm 2.5$ & $3.7 \pm 2.7$ & 0.90 \\
\hline
\end{tabular}

Table 2(b) showed each angle between the subjects who felt pain above the scapula and subjects without pain. There were significant differences in TK and LL between two groups. TK: Thoracic kyphosis LL: Lumber lordosis.

(c)

\begin{tabular}{cccc}
\hline Pain at the interscapula & Normal group $(\mathrm{n}=29)$ & Pain group $(\mathrm{n}=17)$ & $p$ value $(\beta$ value $)$ \\
\hline TK & $27.0 \pm 5.0$ & $30.2 \pm 10.2$ & 0.17 \\
LL & $24.9 \pm 6.7$ & $29.9 \pm 8.5$ & $0.032(0.58)$ \\
Sacral/hip angle & $17.4 \pm 5.6$ & $19.4 \pm 6.7$ & 0.28 \\
Inclination & $3.9 \pm 2.5$ & $3.9 \pm 2.5$ & 0.98 \\
\hline
\end{tabular}

Table 2(c) showed each angle between the subjects who felt pain at the interscapula and subjects without pain. In terms of pain above the scapula, LL significantly increased. TK: Thoracic kyphosis LL: Lumber lordosis. 
increase in thoracic kyphosis [10] [12] [15]. These postures change the position of scapular downward rotation, anterior tilt, and protraction [12]. This malpositioning of the scapula significantly increases the activity of mooring muscles. Compared with a neutral head position, the electromyographic activity of the levator scapula and upper and lower trapezius significantly increased with a forward head position [15] [20]. Moreover, the electromyographic activity of the serratus anterior significantly decreased during loaded isometric shoulder flexion with a forward head position [15]. The upper trapezius is an agonist muscle for upward rotation of the scapula, and the levator scapula is an antagonist. Thus, both the levator scapula and upper trapezius could not relax because both muscles pull each other in a forward head position. Both muscles' insertions are located where the subjects felt pain. These biomechanical reports were consistent with our results. Thus, an increase in thoracic kyphosis easily induces chronic neck and shoulder pain. These results suggest that posture correction, especially correction of thoracic kyphosis, is an effective therapy to improve chronic neck and shoulder pain. We need the further study of investing whether the posture correction improves chronic neck and shoulder pain or not.

In our results, lumbar lordosis had a greater influence for patients with pain above scapula than thoracic kyphosis. Previous papers which assessed the relationship between neck and shoulder pain using SpinalMouse also reported lumber lordosis of patients with neck and shoulder pain was significantly larger than normal [21] Crosbie et al. reported that the lumbar spinal range of motion is insignificant for arm movements and shows no association with any upper limb movement [4]. Although this result was the opposite of our results, many reports have shown that different conditions affect the pattern of arm elevation and the scapulohumeral rhythm [8] [10]-[12] [14] [15] [20]. Compared with a normal head position, elevation of the arm in a forward head position increases the activity of muscles around the neck and shoulder [15] [20]. In this condition, spinal mobility changes. The difference in lumbar lordosis between genders was controversial [13] [22]-[24]. In our investigation, there was no significant difference between genders, although the number of females in the pain group was larger than that in the normal group. Thus, the influence of gender on our results was very small. Sahrmann reported that to achieve a particular range of motion, the body will move through the point of least resistance [25]. The mobility of the thoracic spine was significantly restricted compared with the mobility of the cervical and lumbar spine. Pain decreases the mobility of the thoracic spine and scapula. Lumbar lordosis compensates for thoracic kyphosis to maintain the posture. Therefore, it is easy for lumbar lordosis to be influenced by pain, and lumbar lordosis thus significantly increased in the pain group.

The limitation of this study was that this study was the cross-sectional study. Therefore, we could not confirm whether chronic neck and shoulder pain induce TK and LL increasing or TK and LL increasing induce chronic neck and shoulder pain. However, previous papers reported that themal positioning of the scapula significantly increases the activity of mooring muscles. Compared with a neutral head position, the electromyo graphic activity of the levator scapula and upper and lower trapezius significantly increased with a forward head position [15] [20]. Combined with our results, the modification of supinalsagittal alignment may be effective for improving neck and shoulder pain. We plan to compare spinal alignment change between before and after symptom improvement in the next study.

\section{Conclusion}

Thoracic kyphosis angles and lumbar lordosis angles in a standing posture were significantly larger in the pain group than in the normal group among the younger generation. Thoracic kyphosis in subjects with neck pain or pain above scapula was significantly higher than that in subjects without pain. Lumbar lordosis in subjects with pain above the scapula or interscapula was significantly higher than that in subjects without pain.

\section{Acknowledgements}

The authors thank Takashi Miura for technical support to measure a spinal alignment with the use of the SpinalMouse.

\section{References}

[1] Ministry of Health (2015) Labour and Welfare of Japan. http://www.mhlw.go.jp/toukei/saikin/hw/k-tyosa/k-tyosa10/3-1.html 
[2] Bogduk, N. and Marsland, A. (1988) The Cervical Zygapophysial Joints as a Source of Neck Pain. Spine, 13, 610-617. http://dx.doi.org/10.1097/00007632-198813060-00003

[3] Cloward, R.B. (1959) Cervical Diskography. A Contribution to the Etiology and Mechanism of Neck, Shoulder, and Arm Pain. Annals of Surgery, 150, 1052-1064. http://dx.doi.org/10.1097/00000658-195912000-00013

[4] Crosbie, J., Kilbreath, S.L., Hollmann, L. and York, S. (2008) Scapulohumeral Rhythm and Associated Spinal Motion. Clinical Biomechanics, 23, 184-192. http://dx.doi.org/10.1016/j.clinbiomech.2007.09.012

[5] Ferrari, R. and Schrader, H. (2001) The Late Whiplash Syndrome: A Biopsychosocial Approach. Journal of Neurology, Neurosurgery, and Psychiatry, 70, 722-726. http://dx.doi.org/10.1136/jnnp.70.6.722

[6] Tanaka, Y., Kokubu, S. and Sato, T. (1998) Cervical Radiculopathy and Its Unsolved Problems. Current Orthopaedics, 12, 1-6. http://dx.doi.org/10.1016/S0268-0890(98)90001-9

[7] Wenzel, H.G., Mykletun, A. and Nilsen, T.I. (2009) Symptom Profile of Persons Self-Reporting Whiplash: A Norwegian Population-Based Study (HUNT 2). European Spine Journal, 18, 1363-1370. http://dx.doi.org/10.1007/s00586-009-1106-6

[8] Greenfield, B., Catlin, P.A., Coats, P.W., Green, E., McDonald, J.J. and North, C. (1995) Posture in Patients with Shoulder Overuse Injuried and Healthy Individuals. Journal of Orthopaedic \& Sports Physical Therapy, 21, 287-295. http://dx.doi.org/10.2519/jospt.1995.21.5.287

[9] Haughie, L.J., Fiebert, I.M. and Roach, K.E. (1995) Relationship of Forward Head Posture and Cervical Backward Bending to Neck Pain. Journal of Manual \& Manipulative Therapy, 3, 91-97. http://dx.doi.org/10.1179/jmt.1995.3.3.91

[10] Kebaetse, M., McClure, E. and Pratt, N.E. (1999) Thoracic Position Effect on Shoulder Range of Motion, Strength, and Three-Dimensional Scapular Kinematics. Archives of Physical Medicine and Rehabilitation, 80, 945-950. http://dx.doi.org/10.1016/S0003-9993(99)90088-6

[11] Kibler, W.B. and McMullen, J. (2003) Scapular Dyskinesis and Its Relation to Shoulder Pain. Journal of the American Academy of Orthopaedic Surgeons, 11, 142-151.

[12] Lewis, J.S., Green, A. and Wright, C. (2005) Subacrominal Impingement Syndrome: The Role of Posture and Muscle Imbalance. Journal of Shoulder and Elbow Surgery, 14, 385-392. http://dx.doi.org/10.1016/j.jse.2004.08.007

[13] Vialle, R., Levassor, N., Rillardon, L., Templier, A., Skalli, W. and Guigui, P. (2005) Radiographic Analysis of the Sagittal Alignment and Balance of the Spine in Asymptomatic Subjects. The Journal of Bone \& Joint Surgery, 87, 260267. http://dx.doi.org/10.2106/JBJS.D.02043

[14] Finley, M.A. and Lee, R.Y. (2003) Effect of Sitting Posture on 3-Dimensional Scapular Kinematics Measured by SkinMounted Electromagnetic Tracking Sensors. Archives of Physical Medicine and Rehabilitation, 84, 563-568. http://dx.doi.org/10.1053/apmr.2003.50087

[15] Weon, J.H., Oh, J.S., Cynn, H.S., Kim, Y.W., Kwon, O.Y. and Yi, C.H. (2010) Influence of Forward Head Posture on Scapular Upward Rotators during Isometric Shoulder Flexion. Journal of Bodywork and Movement Therapies, 14, 367374. http://dx.doi.org/10.1016/j.jbmt.2009.06.006

[16] Takagishi, K., Hoshino, Y., Ide, J., Sugihara, T., Hata, Y., Sano, H., et al. (2008) Project Research on Katakori. The Journal of the Japanese Orthopaedic Association, 82, 901-911. (In Japanese)

[17] Kellis, E., Adamou, G., Tzilios, G. and Emmanouilidou, M. (2008) Reliability of Spinal Range of Motion in Healthy Boys Using a Skin-Surface Device. Journal of Manipulative and Physiological Therapeutics, 31, 570-576. http://dx.doi.org/10.1016/j.jmpt.2008.09.001

[18] Mannion, A.F., Knecht, K., Balaban, G., Dvorak, J. and Grob, D. (2004) A New Skin-Surface Device for Measuring the Curvature and Global and Segmental Ranges of Motion of the Spine: Reliability of Measurements and Comparison with Data Reviewed from the Literature. European Spine Journal, 13, 122-136. http://dx.doi.org/10.1007/s00586-003-0618-8

[19] Post, R.B. and Leferink, V.J. (2004) Spinal Mobility: Sagittal Range of Motion Measured with the SpinalMouse, a New Non-Invasive Device. Archives of Orthopaedic and Trauma Surgery, 124, 187-192. http://dx.doi.org/10.1007/s00402-004-0641-1

[20] McLean, L. (2005) The Effect of Postural Correction on Muscle Activation Amplitudes Recorded from Cervicobrachial Region. Journal of Electromyography and Kinesiology, 15, 527-535. http://dx.doi.org/10.1016/j.jelekin.2005.06.003

[21] Tsunoda, D., Iizuka, Y., Iizuka, H., Niahinome, M., Kobayashi, R., Ara, T., Yamamoto, A. and Takagishi, K. (2013) Associations between Neck and Shoulder Pain (Called Katakori in Japanese) and Sagittal Alignment Parameters among the General Population. Journal of Orthopaedic Science, 18, 216-219. http://dx.doi.org/10.1007/s00776-012-0341-6

[22] Jackson, R.P. and McManus, A.C. (1994) Radiographic Analysis of Sagittal Plane Alignment and Balance in Standing 
Volunteers and Patients with Low Back Pain Matched for Age, Sex, and Size. A Prospective Controlled Clinical Study. Spine, 19, 1611-1618. http://dx.doi.org/10.1097/00007632-199407001-00010

[23] Jackson, R.P., Kanemura, T., Kawakami, N. and Hales, C. (2000) Lumbopelviclordosis and Pelvic Balance on Repeated Standing Lateral Radiographs of Adult Volunteers and Untreated Patients with Constant Low Back Pain. Spine, 25, 575-586. http://dx.doi.org/10.1097/00007632-200003010-00008

[24] Legaye, J., Duval-Beaupere, G., Hecquet, J. and Marty, C. (1998) Pelvic Incidence: A Fundamental Pelvic Parameter for Three-Dimensional Regulation of Spinal Sagittal Curves. European Spine Journal, 7, 99-103. http://dx.doi.org/10.1007/s005860050038

[25] Sahrmann, S.A. (2002) Diagnosis and Treatment of Movement Impairment Syndrome. Mosby, St. Louis, 30-31.

\section{List of Abbreviations}

TK: thoracic kyphosis

LL: lumber lordosis 